\title{
Punctuality Algorithm Based on BP Neural Network PID Control
}

\author{
Yingying Yang, Liangfu Peng*, Demei Peng \\ College of Electrical \& Information Engineering, Southwest Minzu University, Chengdu 610041, China \\ Email: 1306929553@qq.com
}

\begin{abstract}
In the modern information society, high-precision clocks are particularly important in the fields of electric power, communications, aviation, and finance, and have very strict objective requirements in terms of frequency accuracy. Currently, the technology of using GPS satellite clock sources to synchronize local clocks has become one of the mainstream methods for generating high-precision clocks at home and abroad. The core idea of this technology is to use the satellite clock to tame the local clock. Due to the development and application of $5 \mathrm{G}$, the accuracy of the system's punctuality have higher requirements, Through analysis, it is found that the combination of BP neural network and PID control can be used to optimize the control of the constant temperature crystal oscillator and improve the precision of punctuality. Finally, the simulation results show that the method has a significant effect in improving the accuracy of punctuality.
\end{abstract}

Keywords: time keeping system; PID control; back propagation (BP) neural network; crystal oscillator taming.

\section{Introduction}

The timekeeping system uses the satellite navigation system to send out a high-precision second pulse signal as the time reference, and uses the satellite navigation system's long-term stability of the second pulse and the short-term stability of the crystal oscillator to complement each other to achieve the purpose of precise time.

The crystal taming technology is the main corrective measure to eliminate the influence of frequency drift on the crystal frequency accuracy. Use a high-precision crystal oscillator to track and correct the GPS second pulse in real time, so that the time information output by the crystal oscillator is constantly close to the GPS time. This is the taming technology of the crystal oscillator.

This article combines ordinary PID control and forward neural network BP neural network to tame the VCOCXO, which solves the limitation that ordinary PID control cannot adjust the clock error in real time and improves the accuracy of punctuality.

\section{Theoretical Analysis}

\subsection{Punctuality Theory}

The main purpose of punctuality is to keep the accuracy of the local frequency standard at the same accuracy as the GPS second pulse signal. The high precision crystal oscillator continuously track the GPS PPS signal, by frequency correction of the crystal is gradually locked to the time information coincides with the GPS time information, thereby achieving punctuality.

\subsection{Factors Affecting Punctuality}

Short-term VCOCXO(Voltage control-oven controlled crystal oscillator, combination of oven controlled crystal oscillator and voltage controlled crystal oscillator) clock signal random error is very small, can be considered absolutely accurate, due to the influence of objective factors such as aging rate, temperature, humidity, and voltage, large random errors will accumulate in a long time. This error is the most important factor affecting the crystal. 


\subsection{Crystal Oscillator Tame}

Crystal tame is the use of long-term stability of the GPS signal the long-term frequency drift of the crystal can be calibrated to achieve local frequency standard signal synchronized with the GPS.

Use GPS 1PPS as the reference standard, compare the phase with the local frequency, and then through the taming algorithm and D/A conversion, the adjustment value of the crystal is obtained to change the crystal frequency.

\section{Punctual System Design}

In order to keep the VCOCXO signal and GPS standard time signal in high-precision synchronization, according to the generation principle of high-precision synchronous clock-keeping, a voltage-controlled oscillator phase-locked loop circuit scheme is designed. It consists of GPS receiver, VCOCXO, phase detector, loop filter, regulator, DAC and other components.

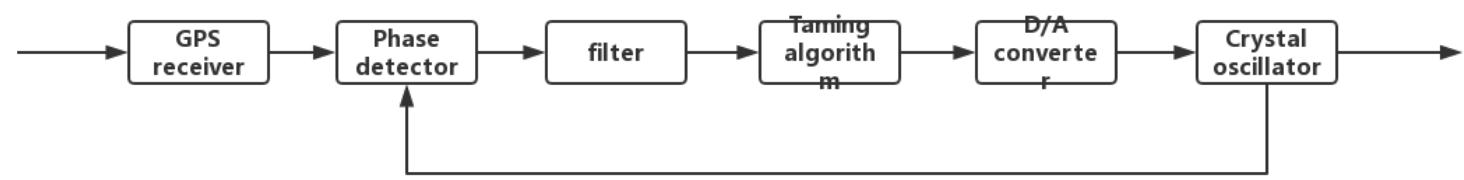

Figure 1. System flow

The GPS 1PPS clock pulse is compared with the VCOCXO $1 \mathrm{~Hz}$ signal in phase, and then the phase difference is processed by Kalman filtering and crystal taming to generate an adjustable control signal, so that the VCOCXO $1 \mathrm{~Hz}$ signal and the GPS 1PPS signal are in the same frequency and phase.

\section{Research on Taming Algorithm}

\subsection{PID Control Algorithm}

The PID controller does not need to know the precise mathematical model of the controlled object and the structure and parameters of the controlled object. It only needs to adjust the three parameters of its proportional coefficient, integral constant and differential constant.

\subsubsection{Analog PID control algorithm}

PID control is a linear control. The input is the signal error of the system. The output $u(t)$ of the controller is obtained through the proportional, integral and differential superposition processing of the error, and then acts on the controlled object to make the actual output consistent with the given value.

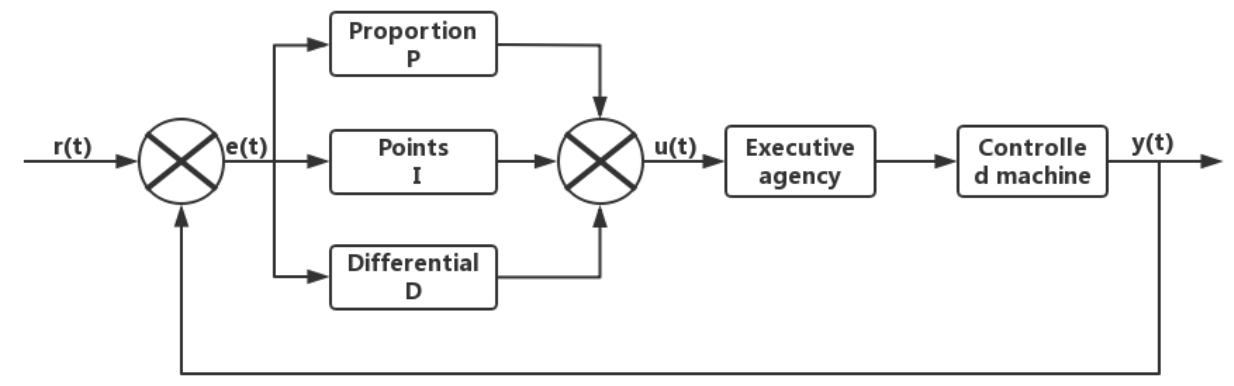

Figure 2. PID control principle diagram

Mathematical expression: 


$$
u(t)=K_{p}\left[e(t)+\frac{1}{T_{i}} \int_{0}^{t} e(t) d t+T_{d} \frac{d e(t)}{d t}\right]
$$

$K_{p}, T_{i}$, Td are the proportional link coefficient, the integral link time constant, and the differential link time constant respectively.

\subsubsection{Position-based digital PID control algorithm}

Digital PID control discretizes time. Assuming that the sampling time is $T, t=K T$ represents the sampling value at $K$ times.

$$
\begin{gathered}
\int_{0}^{t} e(t) d t=T \sum_{j=0}^{k} e(j T)=T \sum_{j}^{k} e(j) \\
\frac{d e(t)}{d t}=\frac{e(k T)-e[(k-1) T]}{T}=\frac{e(k)-e(k-1)}{T}
\end{gathered}
$$

Substitute formula (2), (3) into formula (1)

$$
u(k)=K_{p} e(k)+K_{p} \frac{T}{T_{i}} \sum_{j}^{k} e(j)+K_{p} \frac{T_{d}}{T}[e(k)-e(k-1)]
$$

$K_{i}=K_{p} T / T_{i}, K_{d}=K_{p} T / T_{d}$ are the coefficient of the integral link and the coefficient of the differential link respectively, $k$ is the sample number, $u(k)$ is the output of the computer processor at the $k$ th time; $e(k)$ and $e(k-1)$ are the deviation values at time $k$ and $k-1$, respectively.

Finally, the expression of positional PID control is obtained

$$
u(k)=K_{p} e(k)+K_{i} \sum_{j=0}^{k} e(j)+K_{d}[e(k)-e(k-1)]
$$

Since $u(k)$ is a full-value output, it is necessary to know the error value $e(k)$ between the current time $(k)$ and the previous time $(k-1)$, and the error value is always accumulating, which makes the calculation amount large.

\subsubsection{Incremental digital PID control algorithm}

In order to solve the above problems, the incremental PID control algorithm is generally used in practice, and the output of the incremental digital controller is the increment $\Delta u(k)$ of the control quantity. The prerequisite for the control system to be suitable for the use of incremental PID control algorithm is that the variable input by the actuator is the increment $\Delta u(k)$ of the control quantity.

$$
\begin{gathered}
\Delta u(k)=u(k)-u(k-1) \\
\Delta u(k)=K_{p}[e(k)-e(k-1)]+K_{i} e(k)+K_{d}[e(k)-2 e(k-1)+e(k-2)] \\
=K p \Delta e(k)+K i e(k)+K d[\Delta e(k)-\Delta e(k-1)]
\end{gathered}
$$

The formula (7) is the mathematical model of the incremental PID control algorithm. The incremental PID algorithm does not need to be accumulated. You only need to know the error of this time and the previous two times to obtain $\Delta u(k)$, so the influence of error calculation is small.

\subsection{BP Neural Network PID Control}

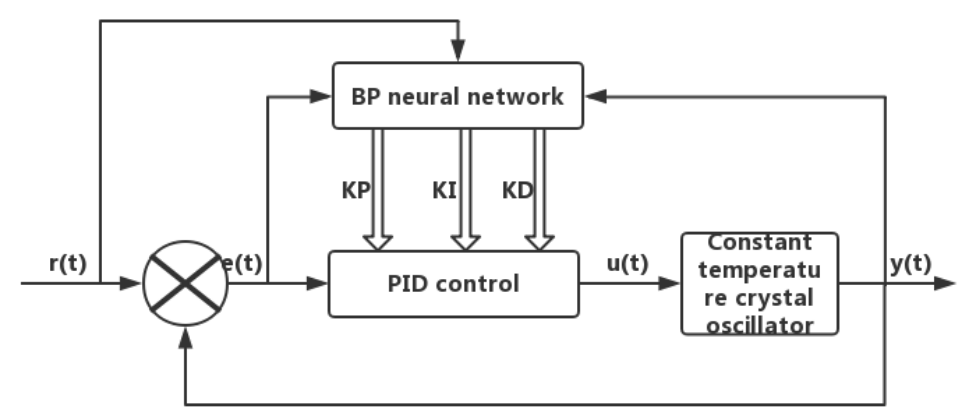

Figure 3. BP neural network PID control principle diagram 
Because the punctual system has the characteristics of nonlinearity and time-variability, the parameters $K_{p}, K_{i}, K_{d}$ of the traditional PID controller do not change with the actual situation, so it cannot achieve high-precision control. The neural network has self-learning ability to adaptively adjust to complex systems. In the PID control method based on the error back propagation method (BP) neural network, the BP neural network can adjust the weighting coefficient through its own self-learning ability, and then automatically and effectively adjust the control parameters of the PID controller. Obtain the optimal control parameters $K_{p}, K_{i}, K_{d}$, so that the system performance indicators reach the ideal state.

\subsubsection{BP neural network structure}

The BP neural network is a multi-layer feedforward neural network trained according to the error back propagation algorithm. The neural network structure includes an input layer, a hidden layer and an output layer. The reverse transmission algorithm can be divided into two parts: forward transmission and reverse feedback. The forward transmission is responsible for calculating the output value layer by layer, and the reverse feedback adjusts the weight of the network layer by layer in the reverse direction according to the output value.

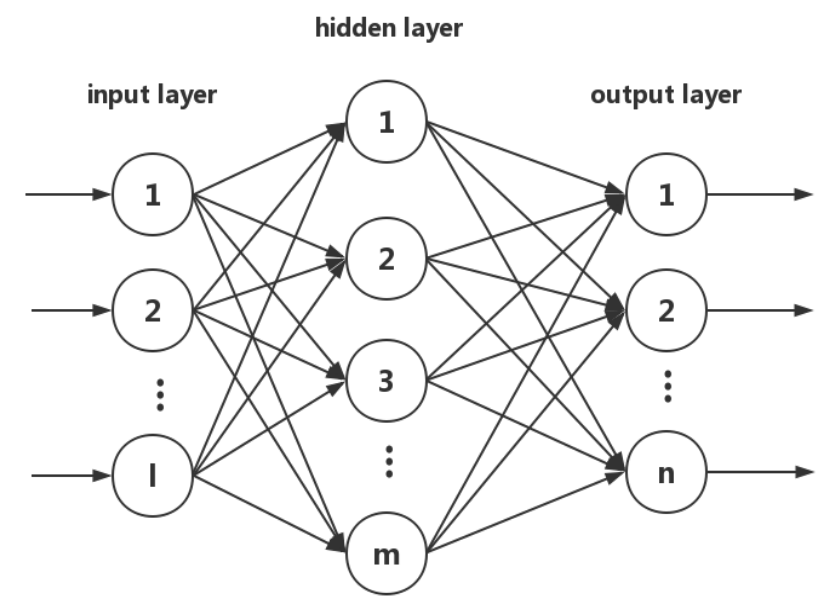

Figure 4. Neural network structure

\subsubsection{BP neural network algorithm}

\section{1) Forward transmission:}

Taking expected value $r(k)$, actual value $y(k)$ and error $e(k)$ as the input of neural network training, the input layer of neural network is expressed as:

$$
O 1_{l}(k)=I 1_{l}(l=1,2,3)
$$

The input and output of the hidden layer of the network are expressed as:

$$
\begin{gathered}
I 2_{m}(k)=\sum_{l=1}^{3} W 2_{l m} O 1_{l} \\
O 2_{m}(k)=f\left(I 2_{m}\right)=f\left(\sum_{l=1}^{3} W 2_{l m} O 1_{l}\right) \quad(m=1,2, \ldots, q)
\end{gathered}
$$

Among them, $W 2_{l m}$ is the weight of the hidden layer, $I 2_{m}$ is the hidden layer input, and $O 2_{m}$ is the hidden layer output.

In a neural network, the number of nodes in the input layer and output layer is known, and the number of nodes in the hidden layer is uncertain. The number of hidden layer nodes has an impact on the performance of the neural network. According to the empirical formula, the number of hidden layer nodes can be determined:

$$
q=\sqrt{a+b}+c
$$


$q$ is the number of hidden layer nodes, $a$ is the number of input layer nodes, $b$ is the number of output layer nodes, and $c$ is an adjustable constant between 1 and 10 .

The Sigmoid function has the characteristics of positive symmetry and negative symmetry, as the activation function of hidden layer neurons:

$$
f(x)=\tanh (x)=\frac{e^{x}-e^{-x}}{e^{x}+e^{-x}}
$$

Input and output of the network output layer:

$$
\begin{gathered}
I 3_{n}(k)=\sum_{m=1}^{q} W 3_{m n} O 2_{m} \\
O 3_{n}(k)=g\left(I 3_{n}\right)=g\left(\sum_{m=1}^{q} W 3_{m n} I 2_{m}\right) \quad(n=1,2,3)
\end{gathered}
$$

The nodes of the output layer of the network neuron correspond to the PID controller parameters $K_{p}, K_{i}$ $K_{d}$, namely $O 3_{1}=K_{p}, O 3_{2}=K_{i}, O 3_{3}=K_{d}$. Since $K_{p}, K_{i}, K_{d}$ are non-negative numbers, and the Sigmoid function has non-negative characteristics, it can be selected as the activation function of the output neuron:

$$
g(x)=\frac{1}{2}(1+\tanh (x))=\frac{e^{x}}{e^{x}+e^{-x}}
$$

\section{2) Backward feedback:}

Select performance index function:

$$
E(k)=\frac{1}{2}[r(k)-y(k)]^{2}
$$

According to the gradient descent method, the weighting coefficient of the neural network is adjusted, that is, according to the given network performance index function $E(k)$, the negative gradient direction of the network weighting coefficient is searched and adjusted, and a global minimum inertia term is added to speed up the search convergence.

The inertia term is:

$$
\Delta W 3_{m n}(k)=-\eta \frac{\partial E(k)}{\partial W 3_{m n}}+\alpha \Delta W 3_{m n}(k-1)
$$

In the formula, the network learning rate is represented by the symbol $\eta$, and the inertia coefficient is represented by the symbol $\alpha$.

$$
\begin{gathered}
\frac{\partial E(k)}{\partial W 3_{m n}}=\frac{\partial E(k)}{\partial y(k)} \cdot \frac{\partial y(k)}{\partial u(k)} \cdot \frac{\partial u(k)}{\partial O 3_{n}(k)} \cdot \frac{\partial O 3_{n}(k)}{\partial I 3_{n}(k)} \cdot \frac{\partial I 3_{n}(k)}{\partial W 3_{m n}} \\
\frac{\partial I 3_{n}(k)}{\partial W 3_{m n}}=O 2_{m}(k)
\end{gathered}
$$

The sign function $\operatorname{sgn}\left(\frac{\partial y(k)}{\partial u(k)}\right)$ is used to approximately replace the unknown quantity $\frac{\partial y(k)}{\partial u(k)}$ by adjusting the learning rate $\eta$ to compensate for the inaccurate effects of the calculation.

Combining all the above formulas can be obtained:

$$
\begin{gathered}
\Delta W 3_{m n}(k)=\alpha \Delta W_{m n}(k-1)+\eta e(k) \operatorname{sgn}\left(\frac{\partial y(k)}{\partial u(k)}\right) \frac{\partial u(k)}{\partial O 3_{n}(k)} g^{\prime}\left(I 3_{n}(k)\right) O 2_{m}(k) \\
\delta 3_{n}=e(k) \operatorname{sgn}\left(\frac{\partial y(k)}{\partial u(k)}\right) \frac{\partial u(k)}{\partial O 3_{n}(k)} g^{\prime}\left(I 3_{n}(k)\right) \quad(n=1,2,3) \\
\Delta W 3_{m n}(k)=\alpha \Delta W_{m n}(k-1)+\eta \delta 3_{n} O 2_{m}(k)
\end{gathered}
$$

$\delta 3_{n}$ is the error assumed on the neurons in the output layer.

Hidden layer network learning algorithm obtained weighting coefficients is:

$$
\delta 2_{m}=\sum_{n=1}^{3} W 3{ }_{m n}(k) \delta 3_{n} f^{\prime}(I 2 m(k))
$$




$$
\Delta W 2 \operatorname{lm}(k)=\alpha W 2{ }_{l m}(k-1)+\eta \delta 2 m O 1_{l}(k)
$$

$\delta 3_{m}$ is the error assumed on the neurons in the output layer.

Finally, the establishment of BP neural network is completed by setting parameters such as learning rate and inertia coefficient.

\section{Analysis of Simulation Results}

In order to verify the effect of the BP neural network PID control method in the punctual control system, Matlab simulation software was used to simulate the BP neural network PID control.

Settings for BP neural network:

Using a 3-5-3 neural network structure, learning rate $\eta=0.25$, inertia coefficient $\alpha=0.05$, sampling interval $t=1$ day, a total of 300 days. The weights $W 2_{l m}$ from the input layer to the hidden layer are set to several random numbers in the interval $\left[-1 \times 10^{-5}, 1 \times 10^{-5}\right]$, and the weights $W 3_{m n}$ from the hidden layer to the output layer are set to the interval $\left[-1 \times 10^{-3}, 1 \times 10^{-3}\right]$ Random number between, using logarithmic model plus random number to imitate the frequency change of real crystal oscillator. The input signal for tracking is $10 \mathrm{MHz}$, and the crystal oscillator is a constant temperature crystal oscillator of $10 \mathrm{MHz}$. The simulation results are shown in Figure 5, 6.

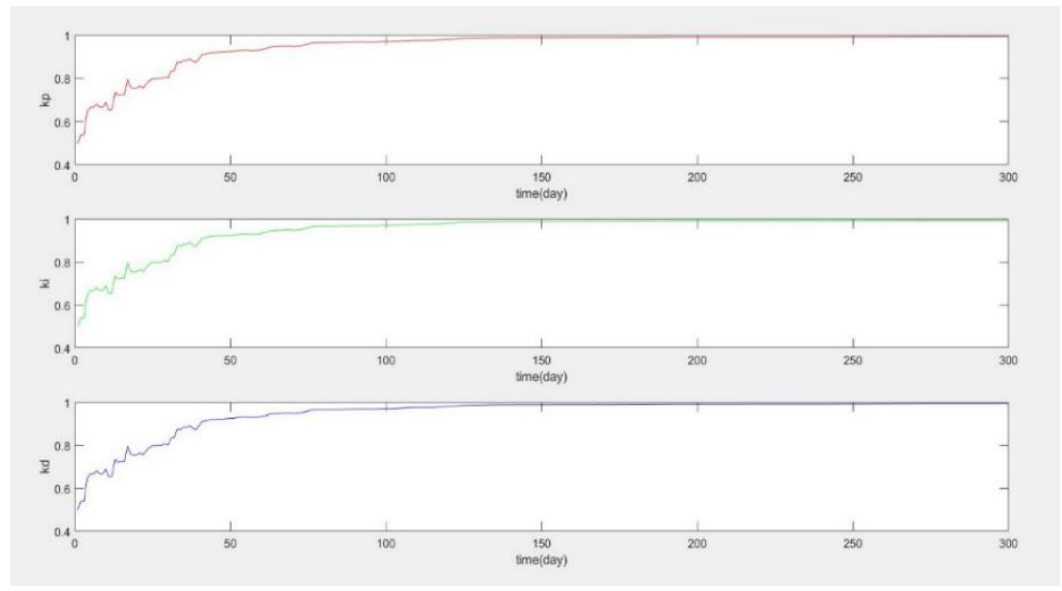

Figure 5. BP neural network PID control parameters

Figure 5 shows that the neural network has been adaptively adjusting PID control parameters for 300 days.

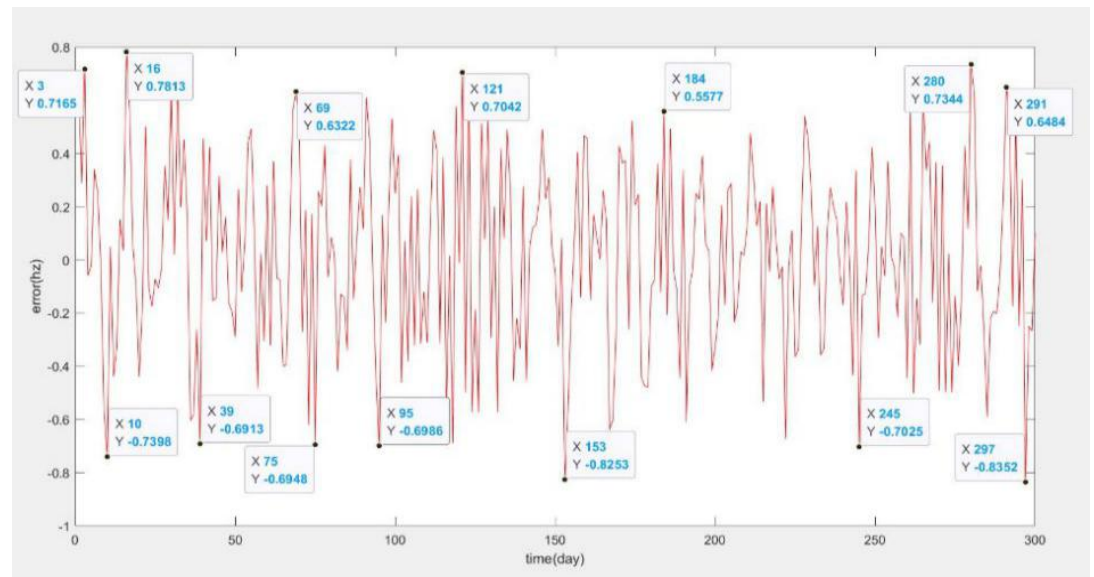

Figure 6. Input and output error of BP neural network PID control 


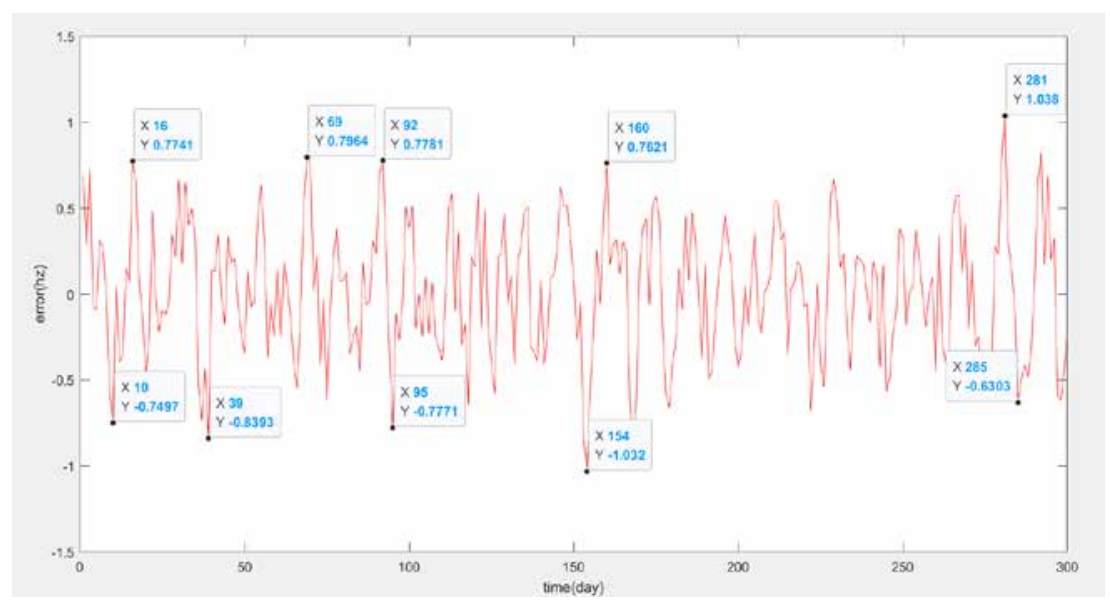

Figure 7. Input and output error of BP ordinary PID control

Figure 6 and 7 seen comparison, the neural network PID control error is at most $0.8352 \mathrm{~Hz}$, generally not exceeding $0.8 \mathrm{~Hz}$, accuracy in time keeping 0.08us;However, the Kp, Ki, and Kd of ordinary PID control are fixed values and cannot be changed in real time to reduce the daily clock error. Therefore, the error may exceed $1 \mathrm{~Hz}$ on a certain day.It can be seen that the BP neural network PID control has a good taming effect on VCOCXO.

\section{Conclusion}

The comparison of the simulation results between ordinary PID control and BP neural network PID control shows that BP neural network regulation PID control is better than ordinary PID control. In the taming of VCOCXO, the traditional PID control parameters $\mathrm{Kp}$, Ki, Kd cannot be adjusted by themselves and need to be determined based on experience, which will waste time. But the BP neural network PID control will adaptively adjust the three control parameters Kp, Ki, Kd during the online learning process of the BP neural network. In addition, the BP neural network has powerful real-time performance and can be controlled in time when deviations are detected. In summary, the BP neural network PID control is more effective in VCOCXO control. Therefore, the control algorithm has high value and application prospects for improving the accuracy of synchronized clock sources in terms of clock taming.

Acknowledgements. The work of this paper is supported by the Southwest Minzu University Graduate Innovative Research Project (Master Program CX2020SZ93). A special acknowledgement should give to Southwest Minzu University for its experimental conditions and technical support.

\section{References}

1. Zhang Yongzhen, Su Hansong, Liu Gaohua. PID controller parameter adjustment based on BP neural network [J]. Journal of Nankai University (Natural Science Edition), 2018, 51 (3): 28-32.

2. Xu Yuchao. Research on Heating Control System of Diamond Press Based on BP Neural Network PID[D]. Guilin University of Electronic Technology, 2017.

3. Sheng Zhongbiao. BP Neural Network Principles and Matlab Simulation[J]. Journal of Wei-nan Teachers University, 2008, 23(5): 65-67.

4. Huang Xiang, Jiang Daozhuo. A High Accuracy Time Keeping Scheme Based oil GPS[J]. Automation of Electric Power Systems, 2010, 34(18): 74-77.

5. Feng Xueyang. Research and Implementation of the Taming and Adaptive Holding Technology of Constant Temperature Crystal Oscillator Based on GPS Second Pulse[D]. School of Communication \& Information Engineering, 2014. 
6. Wang Hongjian, Wang Ling, Huang Wende, Liu Zhijian. Adaptive correction algorithm for high precision time maintenance of OCXO[J]. Transducer and Microsystem Technologies, 2018, 37(4): 132-135.

7. Wang Yan hui. The method and realization of BDS/GPS pseudorange timing and satellite monitoring[D]. Liaoning University of Engineering and Technology, 2017.

8. Zhang Haibin. Beidou punctual and timing system design[D]. San Xia University, 2015.

9. Wang Hongjian. Adaptive correction algorithm for high precision time maintenance of oven controlled crystal oscillator[D]. Hu Nan University, 2017.

10. Zhu Shaohua, Lu Lin, Zhang Bao-fu, Zhu Yong, Wu Chuanxin. Design and realization of a disciplined clock module used in time transfer system with optical fiber[J]. Journal of Time and Frequency, 2011, 34(2): 106-111.

11. Yuan Jianping, Shi Yiping, Jiang Peng, Jiang Yu, Jia Rijing, Yao Deliang. Design of Wind Pendulum Control System Based on BP Neural Network PID Algorithm[J]. Measurement and Control Technology, 2018, 37(11): 144-147.

12. Zhang Yongzhen, Su Hansong, Liu Gaohua, Liao zelong. Parameter Adjustment of PID Controller Based on BP Neural Network[J]. Acta Scientiarum Naturalium Universitatis Nankaiensis, 2018, 51(3): 26-30.

13. Wang Jun, Wang Lei, He Xin. The Study of High Accuracy Time Keeping Based on FPGA When Navigation Satellite Losing Connection[J]. Chinese Journal of Electron Devices, 2016, 39(1): 140-143.

14. Zhao Zhongkun, Song Zhi, Xue Yanbing. Design of a 915MHz Signal Generator Based on PLL Principle[J]. Journal of Telemetry, Tracking and Command, 2017, 38(1): 68-74.

15. Mao Zhendong. Study on Principle and Structure of a Digital-Analog Hybrid Phase Locked Loop[J]. MICROPROCESSORS, 2019, 17(5): 17-20.

16. Hou Yanhong. Network synchronous clock module design and implementation based on STM32F107[D]. Xi'an University of Technology, 2017.

17. Zhou Zheng, Chu Ying-jun. Time Synchronization Network for Power Grid[J]. Frequency and Time Synchronization, 2014, 6(9): 76-79. 